\title{
Preoperative chemoradiotherapy followed by local excision in clinical T2N0 rectal cancer
}

Young Seob Shin, MD1, Yong sik Yoon, MD, PhD², Seok-Byung Lim, MD, PhD², Chang Sik Yu, MD, PhD², Tae Won Kim, MD, PhD³, Heung Moon Chang, MD, PhD², Jin-hong Park, MD, PhD1, Seung Do Ahn, MD, PhD ${ }^{1}$, Sang-Wook Lee, MD, PhD', Eun Kyung Choi, MD, PhD', Jin Cheon Kim, MD, PhD², Jong Hoon Kim, MD, PhD'

Departments of ${ }^{1}$ Radiation Oncology, ${ }^{2}$ Surgery, and ${ }^{3}$ Medical Oncology, Asan Medical Center, University of Ulsan College of Medicine, Seoul, Korea

Purpose: To investigate whether preoperative chemoradiotherapy (PCRT) followed by local excision (LE) is feasible approach in clinical T2NO rectal cancer patients.

Materials and Methods: Patients who received PCRT and LE because of clinical T2 rectal cancer within $7 \mathrm{~cm}$ from anal verge between January 2006 and June 2014 were retrospectively analyzed. LE was performed in case of a good clinical response after PCRT. Patients' characteristics, treatment record, tumor recurrence, and treatment-related complications were reviewed at a median follow-up of 49 months.

Results: All patients received transanal excision or transanal minimally invasive surgery. Of 34 patients, 19 patients (55.9\%) presented pathologic complete response (pCR). The 3-year local recurrence-free survival and disease free-survival were 100.0\% and 97.1\%, respectively. There was no recurrence among the patients with pCR. Except for 1 case of grade 4 enterovesical fistula, all other late complications were mild and self-limiting.

Conclusion: PCRT followed by an LE might be feasible as an alternative to total mesorectal excision in good responders with clinical T2NO distal rectal cancer.

Keywords: Radiotherapy, Rectal neoplasms, Neoadjuvant therapy, Transanal endoscopic microsugery

\section{Introduction}

Total mesorectal excision (TME) is now a mainstay of treatment for localized rectal cancer. However, TME involves the risk of significant complications such as perioperative morbidity, permanent stoma, and impairment of defecation, as well as impaired urinary and sexual functions $[1,2]$. Thus, a local excision (LE) such as a transanal excision (TAE) and transanal endoscopic microsurgery is on the rise as the alternative to TME in rectal cancer to avoid these complications.

However, LE alone is recommended only in clinical T1NO rectal cancer without high-risk features, such as poorly differentiated histology, lymphovascular invasion, and perineural invasion $[3,4]$. The oncologic outcome of LE in more advanced rectal cancer is known to be inferior to that of TME $[5,6]$.

Received 12 July 2016, Revised 10 August 2016, Accepted 30 August 2016.

Correspondence: Jong Hoon Kim, MD, PhD, Department of Radiation Oncology, Asan Medical Center, University of Ulsan College of Medicine, 88 Olympic-ro 43-gil, Songpa-gu, Seoul 05505, Korea. Tel: +82-2-3010-4434, Fax: +82-2-3010-6950, E-mail: jhkim2@amc.seoul.kr

(c) This is an Open Access article distributed under the terms of the Creative Commons Attribution Non-Commercial License (http://creativecommons.org/ licenses/by-nc/4.0/) which permits unrestricted non-commercial use, distribution, and reproduction in any medium, provided the original work is properly cited.

www.e-roj.org 
In locally advanced rectal cancer, preoperative chemoradiotherapy (PCRT) have been proven to decrease the local recurrence, improve the survival, and is now widely used as a standard treatment [7-9]. Additionally, considerable patients experience downstaging following PCRT. Several studies have reported that approximately $20 \%$ patients had a complete response (CR) to PCRT, and these patients had a favorable oncologic outcome [10-12].

These results aroused interest in using LE instead of TME in the patients who have a good response to PCRT. Recently, several retrospective and prospective single-arm trials reported similar local control and survival of LE to TME [1317]. However, the evidence of LE is still limited and there is a concern about possible incomplete oncologic treatment to use LE in all good responders to PCRT [18]. Further investigation is needed to demonstrate the oncologic safety of $L E$, and to find the appropriate candidate to the treatment.

Therefore, we analyzed data from our institution to investigate whether clinical T2NO rectal cancer patients is appropriate candidate to PCRT and LE.

\section{Materials and Methods}

\section{Patients}

We retrospectively reviewed rectal cancer patients who received PCRT and full-thickness LE for curative intent between January 2006 and June 2014. All patients were required to meet the following criteria for the entry into the study: 1) pathologically confirmed cT2 rectal cancer in transanal ultrasonography (TUS) and/or magnetic resonance imaging (MRI); 2) no evidence of regional lymph node or distant metastasis; 3 ) middle to distal rectal cancer within 7 $\mathrm{cm}$ from the anal verge; and 4) good clinical response to PCRT (CR or near CR). Of the 91 patients who received PCRT and LE, 34 patients were suitable to inclusion criteria.

The pretreatment evaluation included the history, physical examination, complete blood counts (CBC), biochemical profiles, serum carcinoembryonic antigen (CEA) levels, a colonoscopy with biopsy, pelvic MRI, chest computed tomography (CT), TUS, and ${ }^{18} \mathrm{~F}$-fluorodeoxyglucose positron emission tomography-CT.

\section{Treatments}

A median 50.0 Gy (range, 43.2 to $51.0 \mathrm{~Gy}$ ) of preoperative radiotherapy was administered to all patients in 1.8-2.0Gy daily fractions. The most common dose scheme was 44.0 Gy to the entire pelvis, followed by a 6.0-Gy boost to the primary tumor in a 2.0-Gy daily fraction. Radiotherapy was delivered through three fields (posterior to anterior and two laterals) using the megavoltage beam (6 or $15 \mathrm{MV}$ ) from a linear accelerator (Varian Medical systems, Palo Alto, CA, USA). The clinical target volume encompassed the primary tumor, perirectal adipose tissue, internal iliac, and presacral nodes. The superior border of CTV was the bottom of L5 spine and the inferior border was $2 \mathrm{~cm}$ distal to the tumor. PTV was 7 $\mathrm{mm}$ expansion of CTV in radial margins and $10 \mathrm{~mm}$ expansion of CTV longitudinally. All patients received concurrent chemotherapy during radiotherapy. The chemotherapy regimen was oral capecitabine $\left(825 \mathrm{mg} / \mathrm{m}^{2}\right)$ administered twice daily during radiotherapy or two cycles of a 5 -FU $\left(375 \mathrm{mg} / \mathrm{m}^{2} /\right.$ day, for 3 days) bolus with leucovorin during the first and fifth week of radiotherapy.

All patient underwent LE after median 7 weeks (range, 6 to 12 weeks) following the end of preoperative treatment. Conventional TAE or transanal minimally invasive surgery (TAMIS) technique were used. TAMIS used standard laparoscopic instruments for TAE, through a single laparoscopic surgery port introduced into the anal canal. The remaining primary lesion and scar tissue were excised with at least a $1 \mathrm{~cm}$ margin and full depth of the rectal wall. Before surgery, TUS and MRI were performed in all but one of the patients to assess a clinical treatment response. We graded the tumor as good clinical response ( $C R$ or near CR) when echoic abnormal lesion in TUS or residual mass in MRI was not visible, or when only small residual lesion is visible with uncertain tumor viability. LE was performed in patients who were medically unfit for radical surgery, who refused radical surgery, or who were in case of a good clinical response after PCRT. The operation method of the patients with good clinical response was determined under sufficient information about the advantages and disadvantages of LE. Adjuvant chemotherapy was performed in most of the patients $(21 / 34$, $61.8 \%$ ) according to the pathological stage and general medical condition of each patient. The most common regimen was six cycles of a 5 -FU ( $375 \mathrm{mg} / \mathrm{m}^{2} /$ day, for 5 days) bolus with leucovorin.

\section{Evaluation and follow-up}

All patients received a regular examination after surgery, every 3-6 months for the first 2 years, every 6 months for the next 3 years, and annually after that. At each follow-up, a complete history, physical examination, laboratory tests, including serum CEA levels, and abdominopelvic CT were performed. A chest $X$-ray or chest CT was performed alternatively every 6 months. 
Colonoscopy or sigmoidoscopy was alternatively performed every 6 months for 2 years after surgery, and annually for the next 3 years.

During and after the treatment, acute and late toxicities were scored according to common terminology criteria for adverse events version 4.0. Acute toxicities were defined as those that occurred up to 90 days from surgery, and late toxicities were defined as those that occurred after 90 days.

\section{Pathologic examination}

Pathologic stage was determined using the TNM classification system, as recommended by the American joint committee on cancer (AJCC), 7th edition. Evaluation of the tumor response to PCRT in the surgical specimen was performed using the tumor regression grading system by Dworak et al. [19]. Briefly, the tumor response was graded as follows: 1) tumor regression

Table 1. Patients' characteristics $(n=34)$

\begin{tabular}{|c|c|}
\hline Variable & Value \\
\hline Age (yr) & $63.6(36.0-83.0)$ \\
\hline \multicolumn{2}{|l|}{ Gender } \\
\hline Male & $16(47.1)$ \\
\hline Female & $18(52.9)$ \\
\hline \multicolumn{2}{|l|}{ Pretreatment CEA (ng/mL) } \\
\hline$<4$ & $33(97.1)$ \\
\hline$\geq 4$ & $1(2.9)$ \\
\hline Distance from anal verge $(\mathrm{cm})$ & $3(0-7)$ \\
\hline \multicolumn{2}{|l|}{ Operation } \\
\hline TAE & $28(82.4)$ \\
\hline TAMIS & $6(17.6)$ \\
\hline \multicolumn{2}{|l|}{ Histologic grade } \\
\hline Well differentiated & $11(32.4)$ \\
\hline Moderately differentiated & $22(64.7)$ \\
\hline Poorly differentiated & $1(2.9)$ \\
\hline \multicolumn{2}{|l|}{ Resection margin } \\
\hline Positive & $1(2.9)$ \\
\hline Negative & $33(97.1)$ \\
\hline \multicolumn{2}{|l|}{ Lymphovascular invasion } \\
\hline Yes & $0(0)$ \\
\hline No & $34(100)$ \\
\hline \multicolumn{2}{|l|}{ Perineural invasion } \\
\hline Yes & $0(0)$ \\
\hline Indeterminated & $1(2.9)$ \\
\hline No & $33(97.1)$ \\
\hline \multicolumn{2}{|l|}{ Adjuvant chemotherapy } \\
\hline Yes & $21(61.8)$ \\
\hline No & $13(38.2)$ \\
\hline
\end{tabular}

Values are presented as the median (range) or number (\%). CEA, carcinoembryonic antigen; TAE, transanal excision; TAMIS, transanal minimally invasive surgery. grade (TRG) 4, no vital tumor cells were detectable; 2) TRG 3 , only scattered tumor cells in the space of fibrosis with/ without acellular mucin; 3) TRG 2, predominantly fibrosis with scattered tumor cells; 4) TRG 1, predominantly a tumor with significant fibrosis and/or vasculopathy; and 5) TRG 0, no regression.

\section{Statistical analysis}

Continuous variables are presented as mean or median (range) values. Local recurrence-free survival (LRFS), diseasefree survival (DFS) and overall survival (OS) were calculated from the date of the start of treatment to the date of death recurrence, or the last follow-up using the Kaplan-Meier method. Statistical significance was accepted at the $p<0.05$ level. All statistical analyzes were performed using SPSS ver. 18.0 (SPSS Inc., Chicago, IL, USA).

\section{Results}

\section{Patient population and tumor characteristics}

We reviewed 34 patients according to sex, age, clinicopathological characteristics of the tumor, the variables for treatment, tumor recurrence, and survival. The patient characteristics are summarized in Table 1. The mean age was 63.6 years (range, 36.0 to 83.0 years) and 16 patients (47.1\%) were male. The median distance from the anal verge was $3.0 \mathrm{~cm}$ (range, $0-7.0 \mathrm{~cm}$ ). In pretreatment MRI, Iymph nodes of greater than $10 \mathrm{~mm}$ or $5 \mathrm{~mm}$ with suggestive features (heterogeneous signal intensity, and irregular border) were regarded as metastasis. All patients in our study did not have lymph node metastasis in the pretreatment study.

After PCRT, all patients had TUS and 33 had MRI for restaging. The clinical and pathological response to PCRT is presented in Table 2. Twenty-eight patients (82.4\%) underwent TAE, and the others underwent TAMIS. There were 19 patients (55.9\%) that had ypT0 tumors, and 12 (35.3\%) had ypT1 or ypTis tumors. The diagnostic accuracy of MRI and TUS for predicting pathologic complete response ( $\mathrm{pCR}$ ) were calculated. The sensitivity and specificity of MRI were $68.4 \%$ and $35.7 \%$. Those of TUS were $73.7 \%$ and $40.0 \%$. The receiver operating characteristic (ROC) curves of TUS and MRI for pathologic response are presented in Fig. 1.

\section{Survival and recurrence}

The median follow-up duration was 49 months (range, 20 to 121 months). The predicted 3-year and 5-year LRFS were $100.0 \%$ and $94.7 \%$. The 3 -year DFS and OS were $97.1 \%$ and 
100.0\% (Fig. 2).

Two patients experienced a recurrence during the followup period. The summary of the patients who experienced a recurrence is presented in Table 3. Both patients had residual

Table 2. Response to preoperative chemoradiotherapy $(n=34)$

\begin{tabular}{lc}
\hline \multicolumn{1}{c}{ Variable } & No. $(\%)$ \\
\hline Clinical T stage (after PCRT, MRI) & $22(64.7)$ \\
CR/near CR & $11(32.4)$ \\
PR/SD & $1(2.9)$ \\
N/A & \\
Clinical T stage (after PCRT, TUS) & $23(67.6)$ \\
CR/near CR & $11(33.4)$ \\
PR/SD & \\
Pathological T stage & $19(55.9)$ \\
T0 & $12(35.3)$ \\
T1 & $3(8.8)$ \\
T2 & \\
Tumor regression grade & $19(55.9)$ \\
4 & $4(11.8)$ \\
3 & $7(20.6)$ \\
2 & $1(2.9)$ \\
1 & $3(8.8)$ \\
N/A
\end{tabular}

PCRT, preoperative chemoradiotherapy; $M R I$, magnetic resonance imaging; $C R$, complete response; $P R$, partial response; $S D$, stable disease; TUS, transanal ultrasonography; N/A, not applicable.

A

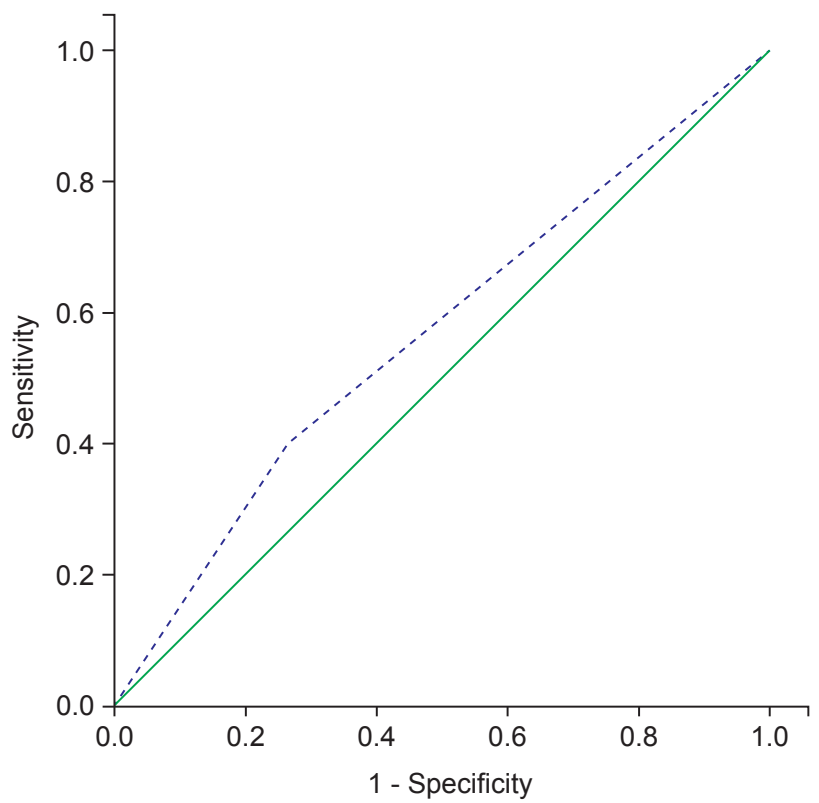

disease after PCRT. One patient developed left internal iliac lymph node recurrence at 40 months from LE. The CT images before, after treatment, and after recurrence are presented in Fig. 3. Although he received a salvage lymph node dissection, he developed multiple retroperitoneal lymph node metastases at the serial follow-up. He is now receiving chemotherapy. The other patient developed liver metastasis at 14 months from the LE. After a partial hepatectomy, he is followed-up without evidence of recurrence for 10 months.

There was no significant difference in the recurrence according to sex, age, tumor differentiation, or adjuvant chemotherapy. Although there was no recurrence among the patients with a pCR, LRFS, DFS and OS between pCR and nonpCR patients were not significantly different (Fig. 4).

\section{Complications during and after treatment}

During PCRT, five severe (G3 or more) complications were observed. Four patients had neutropenia and 1 patient had radiation induced dermatitis, and all patients recovered after PCRT.

For postoperative morbidity, 1 patient had a perianal fistula about a month after LE and received an incision and drainage operation. Other minor complications occurred in 2 patients (5.9\%), including G2 bleeding at the LE site. These two patients were treated with conservative management. There was no

\section{B}

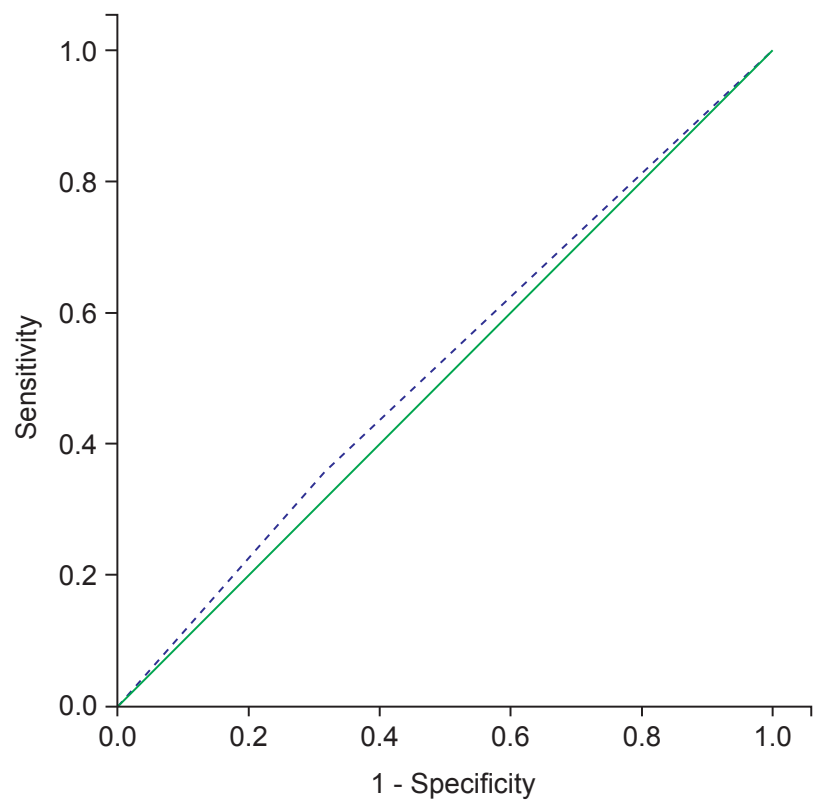

Fig. 1. Receiver operating characteristic curve for predicting pathologic complete response. (A) Transanal ultrasonography and (B) magnetic resonance imaging. 
Table 3. Local and distant recurrence

\begin{tabular}{|c|c|c|c|c|c|c|c|c|c|c|}
\hline & Age/sex & Operation & Site & $\begin{array}{c}\text { ypT } \\
\text { stage }\end{array}$ & $\begin{array}{l}\text { Regression } \\
\text { grade }\end{array}$ & $\begin{array}{c}\text { Resection } \\
\text { margin }\end{array}$ & Adj. CTx & $\begin{array}{c}\text { NED period } \\
(\mathrm{mo})\end{array}$ & $\begin{array}{l}\text { Salvage } \\
\text { treatment }\end{array}$ & Status \\
\hline Patient 1 & Male & TAMIS & c wall) & T2 & Moderate & Negative & Ye & 40 & Salvage OP & AWD \\
\hline Patient 2 & 60/Male & TAE & DM (liver) & T1 & Near total & Negative & Yes & 14 & Salvage OP & NED \\
\hline
\end{tabular}

Adj. CTx, adjuvant chemotherapy; NED, no evidence of disease; TAMIS, transanal minimally invasive surgery; LR, local recurrence; OP, operation; AWD, alive with disease; TAE, transanal excision; DM, distant metastasis.

A

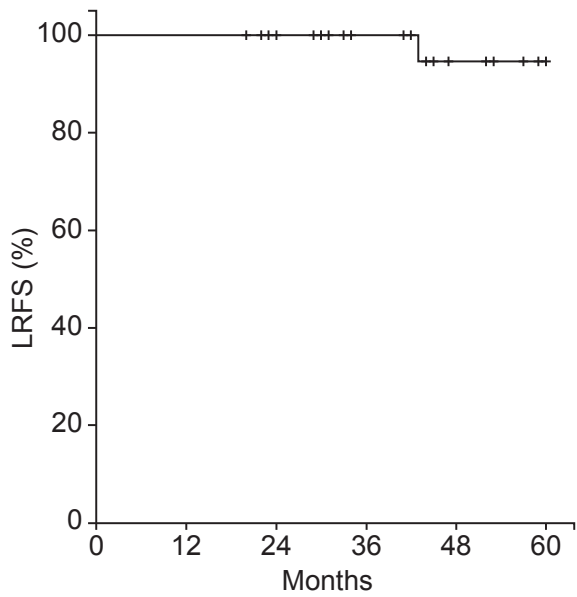

B

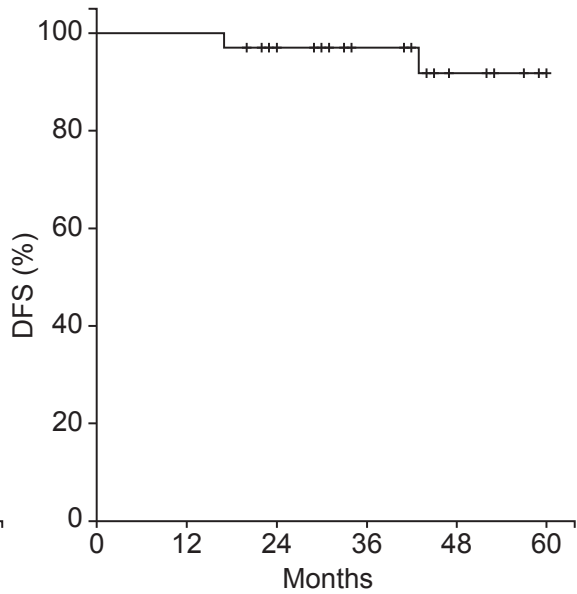

C

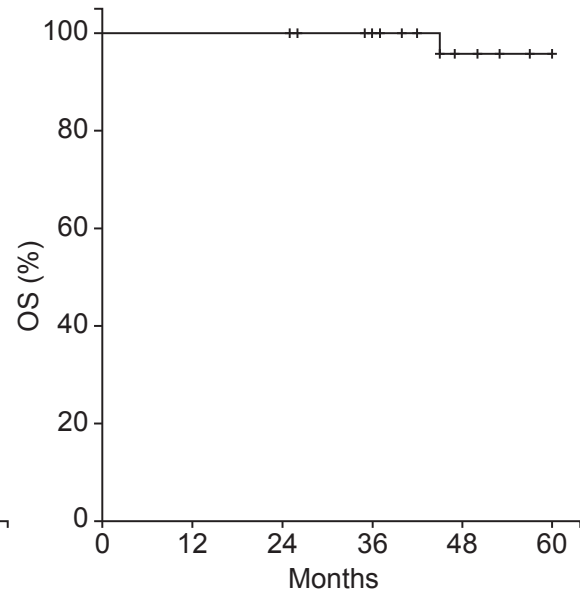

Fig. 2. Survival and recurrence of entire cohort. (A) Local recurrence-free survival (LRFS), (B) disease-free survival (DFS), and (C) overall survival (OS).

postoperative mortality.

During the follow-up, 1 patient received a bowel resection and anastomosis because of an enterovesical fistula after 45 months from LE. Except for this grade 4 toxicity, all other late complications were mild (G2 or less) and well controlled with conservative treatment.

\section{Discussion and Conclusion}

Successful treatment of cancer has resulted in increased demands for the quality of life ( $\mathrm{OOL}$ ) of survivors. After the introduction of TME, local control and survival rates of rectal cancer have markedly improved. However, TME is associated with a decreased OOL [20]. Especially for the patients with distal rectal cancer, abdominoperineal resection (APR) is often needed, which results in a permanent colostomy.

Because there is concern regarding the deterioration of local control, LE has been used only in early rectal cancer $[3,4]$. However, with the advent of preoperative therapy, some patients could be cured or almost cured of cancer before TME. Therefore, there are many questions regarding whether TME is still required in these good responders to PCRT, and LE was suggested as an alternative treatment option.

The results of the present study suggest that we could use LE instead of TME in the selected clinical T2NO rectal cancer patients. Local recurrence, disease-free survival, and OS were good, and only 2 of 34 patients developed recurrence during the follow-up period.

Previous studies reported similar results. Yu et al. [15] demonstrated good disease control and safety of the clinical T2-3NOM0 rectal cancer patients who underwent PCRT and LE. After a median follow-up of 38 months, 3-year DFS was $85.9 \%$. Noh et al. [17] also reported a $82 \%$ of 5 -year DFS rate after PCRT and LE in clinical T2 distal rectal cancer, and suggested LE as a feasible alternative to radical surgery.

For the patients in the present study, the standard approach would be a low anterior resection (LAR) or APR. However, it is reported that about 25\% to 50\% patients presented with LAR syndrome, such as incontinence and soiling after LAR $[21,22]$. In addition, more than half of them experienced a major adverse impact on QOL [20]. Compared to these results, all observed bowel function problems in present study were 

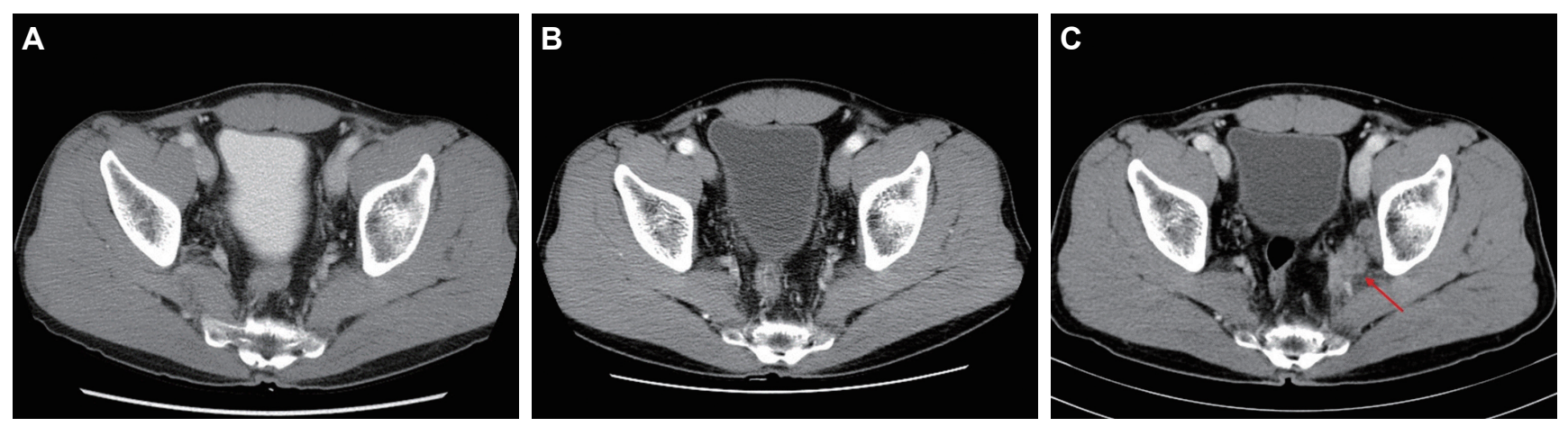

Fig. 3. Computed tomography (CT) images of the patient who experienced a local recurrence: (A) before treatment, (B) after treatment, and (C) after recurrence. CT image shows intermediate density lesion (arrow) adjacent to the left internal iliac vessels.

mild and self-limiting. No patients complained of other late complications known to be associated with TME (e.g., sexual and urinary dysfunction). Furthermore, all patients in the present study exhibited preserved sphincter function throughout the follow-up period.

However, a selection of the appropriate patients for LE is a difficult matter. Because LE alone adversely affected a local control in cT2N0 patients [23], LE should be used in the good responders to PCRT. However, it is difficult to select good responders before the resection of the tumor. To date, none of the non-invasive diagnostic tools is reliable. Neither positron emission tomography (PET), MR, or TUS are sufficient for assessing the response after PCRT. Previous meta-analysis and single center studies demonstrated that the sensitivity of $\mathrm{MRI}$ restaging for complete response was 5\% to 15\%, and the sensitivity of TUS restaging was 35\% to 37\% [24-27]. Similarly, in the present study, both MRI and TUS could not predict a complete response sufficiently. Thus, after the excision, we could elucidate the exact response and decide the course of further treatment.

Because LE leaves most of the mesorectum and all pelvic lymph nodes are unresected, a possible residual disease in these areas is always a problem in using LE. Although all patients in our study did not have lymph node metastasis in pretreatment $M R I$, the accuracy of $M R I$ in predicting lymph node metastasis is insufficient [28]. Park et al. [29] reported that small lymph nodes $(<3 \mathrm{~mm})$ were not detectable in MRI, and $15 \%$ of the lymph nodes contained metastasis in the node-by-node analysis between pretreatment MRI and surgical specimen.

Several studies reported that final pathological T stage was highly correlated with pathological N stage, and it can be used as a predictor of the residual metastatic lymph node $[30,31]$. In the preliminary result of GRECCAR 2 trial [32], good pathologic responders (pathological T0 or $\mathrm{T} 1$ ) showed very low rates of lymph node metastasis compared to bad responders (0\% vs. $15.0 \%$, respectively). Therefore, the pathological response after LE could also reflect the risk of residual disease in regional nodal areas. LE could be used as both a treatment, as well as a diagnostic tool to select the patients with a good response.

The patients presenting with a poor pathological response after LE should receive further treatment, such as TME. Because a standard treatment in clinical T2NO rectal cancer is TME alone, these poor responders could receive unintended overtreatment, PCRT and TME. Therefore, clinicians should confer with the patients sufficiently before starting the treatment.

However, there is no consensus regarding the selection of poor responders following the PCRT. There were different selection criteria, such as pathological T2, pathological T3 stage, or a resection margin invasion in several studies $[13,14,33]$.

In ACOSOG Z6041 prospective phase II trial [13], which reported survival and local control in cT2NO rectal cancer, the patients with an ypT3 tumor or resection margin involvement were recommended to have TME within 6 months. Of the three ypT3 patients, the one who refused additional surgery developed pelvic recurrence. However, the pelvic recurrence rate of ypT2 patients (1/24) was not higher than the ypT1 (1/11) and ypT0/Tis (1/38) patients.

In Polish LE trial [14], which reported the oncologic outcome in cT1N0 to borderline cT2/T3NO rectal cancer patients, good responders were defined as $\mathrm{PCR}$ or $\mathrm{pT} 1$ without unfavorable factors. For the poor responders, immediate conversion to radical surgery was recommended. During the follow-up period, local recurrence was detected in 5 of 63 (7.9\%) of the 
A

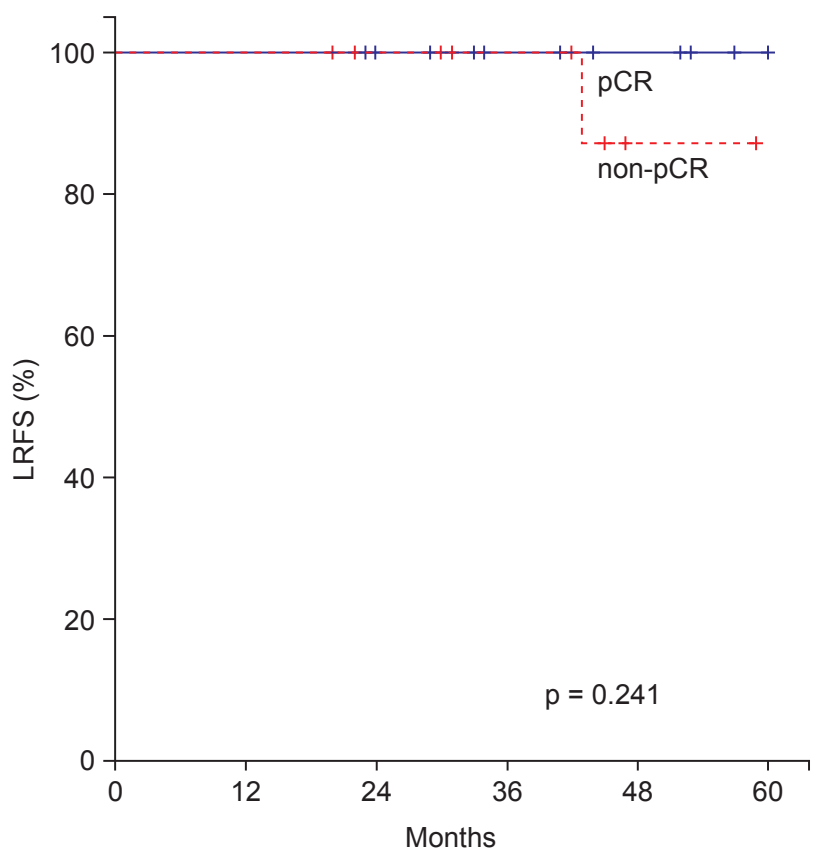

C

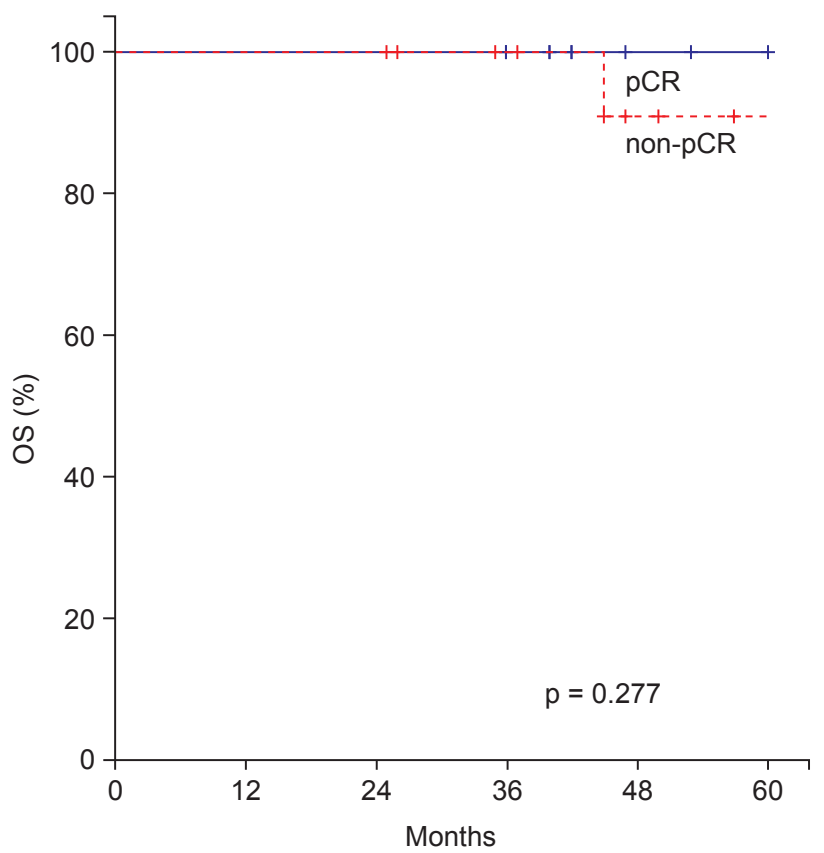

good responders, and 8 of 18 (44.4\%) of the poor responders who did not undergo immediate conversion.

In the present study, the only patient who developed pelvic recurrence was ypT2 $(1 / 3,33.3 \%)$, and none of ypTO/T1 patients recurred in the pelvis. Because of the small sample size of the study, further investigation is required regarding the oncological safety of LE in ypT2 and ypT3 patients.

Although there are few reports about the recurrence
B

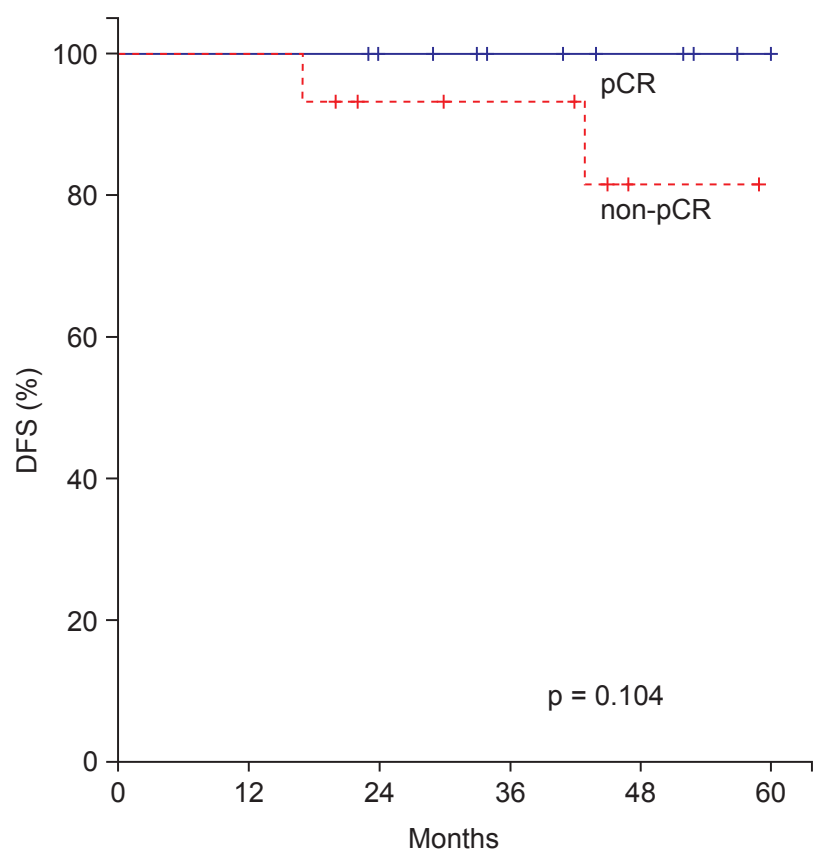

Fig. 4. Survival and recurrence in pathological complete response $(p C R)$ and non-pCR patients. (A) Local recurrence-free survival (LRFS), (B) disease-free survival (DFS), and (C) overall survival (OS).

pattern following PCRT and LE, we think a thorough surveillance of the residual rectum and immediate salvage treatment is important in the patients who received $L E$. Therefore, in our institution, comparing to the patients who received radical resection, more frequent sigmoidoscopy or colonoscopy is conducted owing to the concern about local recurrence. However, in the present study, no patients exhibited a recurrence in the rectal wall. In contrast, in a Polish 
trial [14], all of the local recurrences occurred in the rectal wall with or without the mesorectum. Approximately $50 \%$ of these recurrences were successfully salvaged after APR. Because of this discrepancy in small series, further investigation in a study with a large cohort is required for the pattern of recurrence, surveillance and salvage treatment.

Our study has several limitations. First, because of its retrospective nature, our study has a patient selection bias. Because our clinicians might perform LE in patients with a good response in the evaluation following PCRT, 19 patients (55.9\%) in the present study had pCR. This is fairly high comparing to the patients who received TME in the same period in our institution. 15 of 37 patients (40.5\%) were pCR after PCRT and TME. Prospective trials also reported the proportion of pCR as approximately $41 \%-49 \%$ in cT2NO patients $[13,14]$. Therefore, our study could not represent all clinical T2NO patients, and our results should be limited to good responders. Second, the follow-up period of our study was short. Although most recurrence is known to occur within the first 2 years in rectal cancer, preoperative treatment might delay the development of recurrences [34]. Therefore, more patients in the present study could develop a recurrence in the future.

In conclusion, despite several limitations, our results suggest that LE might be an alternative to TME in clinical T2NO distal rectal cancer patients with good response following PCRT.

\section{Conflict of Interest}

No potential conflict of interest relevant to this article was reported.

\section{References}

1. Chessin DB, Enker W, Cohen AM, et al. Complications after preoperative combined modality therapy and radical resection of locally advanced rectal cancer: a 14-year experience from a specialty service. J Am Coll Surg 2005;200:876-82.

2. Peeters KC, van de Velde CJ, Leer JW, et al. Late side effects of short-course preoperative radiotherapy combined with total mesorectal excision for rectal cancer: increased bowel dysfunction in irradiated patients: a Dutch colorectal cancer group study. J Clin Oncol 2005;23:6199-206.

3. Bach SP, Hill J, Monson JR, et al. A predictive model for local recurrence after transanal endoscopic microsurgery for rectal cancer. Br J Surg 2009;96:280-90.

4. Doornebosch PG, Zeestraten E, de Graaf EJ, et al. Transanal endoscopic microsurgery for $\mathrm{T} 1$ rectal cancer: size matters!
Surg Endosc 2012;26:551-7.

5. Nascimbeni R, Burgart $\amalg$, Nivatvongs $S$, Larson DR. Risk of lymph node metastasis in T1 carcinoma of the colon and rectum. Dis Colon Rectum 2002;45:200-6.

6. Yamamoto $S$, Watanabe $M$, Hasegawa $H$, et al. The risk of lymph node metastasis in T1 colorectal carcinoma. Hepatogastroenterology 2004;51:998-1000.

7. Sebag-Montefiore D, Stephens RJ, Steele R, et al. Preoperative radiotherapy versus selective postoperative chemoradiotherapy in patients with rectal cancer (MRC CRO7 and NCIC-CTG C016): a multicentre, randomised trial. Lancet 2009;373:811-20.

8. Sauer R, Liersch T, Merkel S, et al. Preoperative versus postoperative chemoradiotherapy for locally advanced rectal cancer: results of the German CAO/ARO/AIO-94 randomized phase III trial after a median follow-up of 11 years. J Clin Oncol 2012;30:1926-33.

9. Roh MS, Colangelo LH, O'Connell MJ, et al. Preoperative multimodality therapy improves disease-free survival in patients with carcinoma of the rectum: NSABP R-03. J Clin Oncol 2009;27:5124-30.

10. Martin ST, Heneghan HM, Winter DC. Systematic review and meta-analysis of outcomes following pathological complete response to neoadjuvant chemoradiotherapy for rectal cancer. Br J Surg 2012;99:918-28.

11. Maas M, Nelemans PJ, Valentini $V$, et al. Long-term outcome in patients with a pathological complete response after chemoradiation for rectal cancer: a pooled analysis of individual patient data. Lancet Oncol 2010;11:835-44.

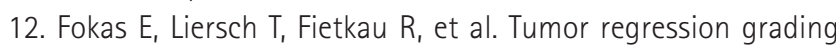
after preoperative chemoradiotherapy for locally advanced rectal carcinoma revisited: updated results of the CAO/ARO/ Al0-94 trial. J Clin Oncol 2014;32:1554-62.

13. Garcia-Aguilar J, Renfro LA, Chow OS, et al. Organ preservation for clinical T2NO distal rectal cancer using neoadjuvant chemoradiotherapy and local excision (ACOSOG Z6041): results of an open-label, single-arm, multi-institutional, phase 2 trial. Lancet Oncol 2015;16:1537-46.

14. Bujko K, Richter P, Smith FM, et al. Preoperative radiotherapy and local excision of rectal cancer with immediate radical re-operation for poor responders: a prospective multicentre study. Radiother Oncol 2013;106:198-205.

15. Yu CS, Yun HR, Shin EJ, et al. Local excision after neoadjuvant chemoradiation therapy in advanced rectal cancer: a national multicenter analysis. Am J Surg 2013;206:482-7.

16. Stipa F, Picchio M, Burza A, Soricelli E, Vitelli CE. Long-term outcome of local excision after preoperative chemoradiation for ypT0 rectal cancer. Dis Colon Rectum 2014;57:1245-52.

17. Noh JM, Park W, Kim JS, et al. Outcome of local excision following preoperative chemoradiotherapy for clinically $\mathrm{T} 2$ distal rectal cancer: a multicenter retrospective study (KROG 
12-06). Cancer Res Treat 2014;46:243-9.

18. Debove C, Guedj N, Tribillon E, Maggiori L, Zappa M, Panis Y. Local excision of low rectal cancer treated by chemoradiotherapy: is it safe for all patients with suspicion of complete tumor response? Int J Colorectal Dis 2016;31:853-60.

19. Dworak O, Keilholz L, Hoffmann A. Pathological features of rectal cancer after preoperative radiochemotherapy. Int J Colorectal Dis 1997;12:19-23.

20. Juul T, Battersby NJ, Christensen $P$, et al. Validation of the English translation of the low anterior resection syndrome score. Colorectal Dis 2015;17:908-16.

21. BregendahI S, Emmertsen KJ, Lous J, Laurberg S. Bowel dysfunction after low anterior resection with and without neoadjuvant therapy for rectal cancer: a population-based cross-sectional study. Colorectal Dis 2013;15:1130-9.

22. Bryant $\mathrm{CL}$, Lunniss $\mathrm{PJ}$, Knowles $\mathrm{CH}$, Thaha MA, Chan CL. Anterior resection syndrome. Lancet Oncol 2012;13:e403-8.

23. You YN, Baxter NN, Stewart A, Nelson H. Is the increasing rate of local excision for stage I rectal cancer in the United States justified?: a nationwide cohort study from the National Cancer Database. Ann Surg 2007;245:726-33.

24. Zhao RS, Wang H, Zhou ZY, Zhou O, Mulholland MW. Restaging of locally advanced rectal cancer with magnetic resonance imaging and endoluminal ultrasound after preoperative chemoradiotherapy: a systemic review and metaanalysis. Dis Colon Rectum 2014;57:388-95.

25. Joye I, Deroose CM, Vandecaveye $\mathrm{V}$, Haustermans K. The role of diffusion-weighted MRI and (18)F-FDG PET/CT in the prediction of pathologic complete response after radiochemotherapy for rectal cancer: a systematic review. Radiother Oncol 2014;113:158-65.

26. Hanly AM, Ryan EM, Rogers AC, et al. Multicenter Evaluation of Rectal cancer Relmaging pOst Neoadjuvant (MERRION) Therapy. Ann Surg 2014;259:723-7.
27. Guillem JG, Ruby JA, Leibold T, et al. Neither FDG-PET Nor CT can distinguish between a pathological complete response and an incomplete response after neoadjuvant chemoradiation in locally advanced rectal cancer: a prospective study. Ann Surg 2013:258:289-95.

28. Al-Sukhni E, Milot L, Fruitman M, et al. Diagnostic accuracy of MRI for assessment of T category, lymph node metastases, and circumferential resection margin involvement in patients with rectal cancer: a systematic review and meta-analysis. Ann Surg Oncol 2012;19:2212-23.

29. Park JS, Jang YJ, Choi GS, et al. Accuracy of preoperative MRI in predicting pathology stage in rectal cancers: node-for-node matched histopathology validation of MRI features. Dis Colon Rectum 2014;57:32-8.

30. Kim DW, Kim DY, Kim TH, et al. Is T classification still correlated with lymph node status after preoperative chemoradiotherapy for rectal cancer? Cancer 2006;106:1694-700.

31. Read TE, Andujar JE, Caushaj PF, et al. Neoadjuvant therapy for rectal cancer: histologic response of the primary tumor predicts nodal status. Dis Colon Rectum 2004;47:825-31.

32. Vendrely $V$, Rullier $E$, Rouanet $P$, et al. Local excision versus total mesorectal excision in patients with good response after neoadjuvant radiochemotherapy for T2-T3 low rectal cancer: preliminary results of the GRECCAR 2 randomized phase 3 trial. Int J Radiat Oncol Biol Phys 2014;90:S20.

33. Yeo SG, Kim DY, Kim TH, et al. Local excision following preoperative chemoradiotherapy-induced downstaging for selected cT3 distal rectal cancer. Jpn J Clin Oncol 2010;40:754-60.

34. Merkel S, Mansmann U, Hohenberger W, Hermanek P. Time to locoregional recurrence after curative resection of rectal carcinoma is prolonged after neoadjuvant treatment: a systematic review and meta-analysis. Colorectal Dis 2011;13:123-31. 